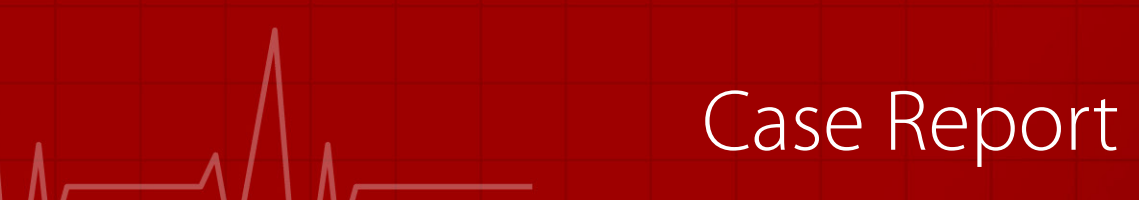

Journal of Emergency Medicine Case Reports

\title{
Hepatotoxicity Caused By Phenyramidol Hydrochloride: A Case Report
}

Hasan Kara, MD; ${ }^{1}$ Aysegul Bayir, MD; Gokhan Gungor, MD; ${ }^{2}$ Ahmet Ak, MD;

'Selçuk University, Faculty of Medicine, Department of Emergency Medicine, Konya, Turkey

${ }^{2}$ Selçuk University, Faculty of Medicine, Department of Gastroenterology, Konya, Turkey

\begin{abstract}
Introduction: Drug-induced liver injury is a common cause of liver dysfunction. Phenyramidol causes a neuronal blocking effect and is used as a moderately potent non-narcotic analgesic that can be accompanied by muscle relaxant activity. In this article, a clinically asymptomatic case of hepatotoxicity is described following the intake of phenyramidol $\mathrm{HCl}$ tablets in a suicide attempt.

Case Report: A 52-year-old female patient was admitted to the emergency department 24 hours after allegedly attempting suicide by ingesting 16 tablets of $400 \mathrm{mg}$ phenyramidol $\mathrm{HCl}$. She displayed no clinical symptoms. An abdominal examination was normal. The results of her first blood test showed: ALT at $58 \mathrm{U} / \mathrm{L}$ and AST at $55 \mathrm{U} / \mathrm{L}$. After 24 hours, ALT and AST were measured at $594 \mathrm{U} / \mathrm{L}$ and $820 \mathrm{U} / \mathrm{L}$, respectively. The patient, whose liver function tests regressed following symptomatic treatment administered in the gastroenterology department, was discharged 5 days later.
\end{abstract}

Conclusion: Drug toxicities can be difficult to diagnose but becomes easier if an agent can be identified that is suspected of causing liver injury. Accordingly, phenyramidol should be considered as a drug that causes hepatotoxicity.

Key Words: Suicide, Phenyramidol, Hepatotoxicity.

\section{Introduction}

The liver metabolizes almost all drugs and toxins that enter the body, resulting in the liver being a common target of drug toxicities. Risk factors for drug-induced liver injury (DILI) include race, age, sex, alcohol intake, liver disease, genetic factors, comorbidities, drug formulations, and host factors. Most drugs feature lipophilic properties that facilitate their absorption through cell membranes. In the liver, drugs undergo biotransformation to obtain hydrophilic properties, which allows for their excretion through bile or urine. The metabolites produced during the biotransformation process are responsible for various toxicities. Medications account for approximately $10 \%$ of all acute hepatitis cases and represent $20 \%-40 \%$ of all fulminant liver failure cases ${ }^{1}$. Hepatocellular injury may be indicated by elevated levels of alanine aminotransferase (ALT) and aspartate aminotransferase (AST), whereas high alkaline phosphatase (ALP) levels typically suggest a cholestatic injury, regardless of bilirubin levels ${ }^{2}$. Phenyramidol HCI, also known as fenyramidol, is an aminopyridine derivative that is used as a moderately potent, non-narcotic muscle relaxant with concomitant analgesic activity. These effects are mediated through the inhibition of interneuronal activity in the spinal cord and brainstem. Phenyramidol metabolism occurs in the liver via glucuronidation. Phenyramidol is conjugated in the liver with glucu- ronic acid and is primarily excreted as phenyramidol glucuronide in urine. A large part of the drug is excreted through the bile and is excreted in the faeces by entering the enterohepatic circulation as free phenyramidol from the small intestine with the effect of bacterial glucuronidase. However, the literature on phenyramidol is limited, and the exact mechanisms underlying phenyramidol-induced liver injury remain unknown. In rare cases, phenyramidol use causes gastrointestinal discomfort (constipation, gastric irritation, itching, sleepiness, weakness, vomiting, and nausea), and some cases of hepatotoxicity have been reported ${ }^{3,4}$. In this article, a clinically asymptomatic case of hepatotoxicity is described following the intake of 16 phenyramidol $\mathrm{HCl} 400 \mathrm{mg}$ tablets in a suicide attempt.

\section{Case Presentation}

A 52-year-old female patient was admitted to the emergency department (ED) 24 hours after allegedly attempting suicide by ingesting 16 tablets of $400 \mathrm{mg}$ phenyramidol HCI. The patient was examined, and the patient displayed a moderate condition and was conscious, lucid, and cooperative. Her vital signs were as follows: systolic blood pressure, 125 $\mathrm{mmHg}$; diastolic blood pressure, $70 \mathrm{mmHg}$; pulse, $87 \mathrm{bpm}$; 
respirations, 16 breaths per minute; and oxygen saturation, $94 \%$ on room air. She displayed no clinical symptoms. In our case, no chronic use of drugs, herbs, or herbal products was reported by the patient. An abdominal examination was normal, with no signs of jaundice, rash, or hepatosplenomegaly. Cardiac auscultation was normal. Electrocardiograph (ECG) evaluation revealed a normal sinus rhythm. The patient's other systemic examinations were normal. The Poison Information Center was consulted for their recommendations, and the patient was admitted to the ED for observation. The results of her first blood test showed: ALT at $58 \mathrm{U} / \mathrm{L}$ and AST at $55 \mathrm{U} / \mathrm{L}$. Other biochemical tests, including serum albumin, bilirubin, creatinine, and potassium, in addition to the complete blood cell count and prothrombin time, were all within normal limits. On ultrasonography, a Grade 2 hepatosteatosis was detected, and the craniocaudal length of the liver was measured at $185 \mathrm{~mm}$ larger than normal. Ultrasonographic examination of the biliary tract was normal. After 24 hours, ALT and AST were measured at $594 \mathrm{U} / \mathrm{L}$ and $820 \mathrm{U} / \mathrm{L}$, respectively. The prothrombin time international normalized ratio (INR) was 1.05 . Viruses that might cause acute hepatitis were screened using serological tests, such as anti-hepatitis A virus (HAV) Ab IgM, hepatitis B surface antigen (HBs Ag), anti-hepatitis $\mathrm{B}$ core antigen $(\mathrm{HBc}) \operatorname{IgM}$, anti-hepatitis $\mathrm{E}$ virus (HEV) $\mathrm{Ab} \operatorname{IgM}$ and $\mathrm{IgG}$, anti-hepatitis $\mathrm{C}$ virus (HCV), and anti-human immunodeficiency virus (HIV) were all negative. The result of the anti-nuclear antibody (ANA) test, as examined using the indirect immunofluorescence assay method, was negative. Thus, other hepatitis etiologies were properly excluded, and the high enzyme levels were attributed to the intake of a high dose of phenyramidol. The patient, who presented with increased liver enzyme levels, was transferred to the gastroenterology department. The time course of liver function tests is presented in Table 1. The patient, whose liver function tests regressed following symptomatic treatment administered in the gastroenterology department, was discharged 5 days later.

Table 1. Time Course of Liver Function Tests

\begin{tabular}{lccccc}
\hline Parameter/ Date & Day 1 & Day 2 & Day 3 & Day 4 & Day 5 \\
\hline ALT (U/L) & 58 & 594 & 441 & 311 & 207 \\
\hline AST(U/L) & 55 & 820 & 299 & 110 & 48 \\
\hline ALP(U/L) & 117 & 193 & 169 & 159 & 141 \\
\hline ALT/ALP & 0,4 & 3 & 2,6 & 1,9 & 1,4 \\
\hline Total Bilirubin (mg/dl) & 1,06 & 2,67 & 2,74 & 1,98 & 0,69 \\
\hline Albumin (g/dl) & 4,2 & 3,9 & 3,9 & 3,6 & 3,6 \\
\hline INR & 0,99 & 1,06 & 1,06 & 0,95 & 0,92 \\
\hline
\end{tabular}

ALT=alanine aminotransferase; $\mathbf{A S T}=$ aspartate aminotransferase;

$\mathbf{A L P}=$ alkaline phosphatase; $\mathbf{I N R}=$ International Normalized Ratio.

\section{Discussion}

Phenyramidol features the properties of a centrally acting oral skeletal muscle relaxant and an analgesic and is generally considered to be an efficacious and well-tolerated drug. However, phenyramidol is also thought to potentially cause hepatotoxicity. The first report of hepatotoxicity in the literature described a 70-year-old male patient who presented with increased liver enzymes during phenyramidol treatment, for whom the enzyme values improved within one week after phenyramidol discontinuation ${ }^{4}$. In a double-blind, randomized controlled study, 7 of 38 patients in the phenyramidol treatment group displayed an increase in liver enzymes that returned to normal levels after a one-week follow-up ${ }^{3}$. In a case report, which represented the first description in the literature of liver enzyme elevation in response to high-dose phenyramidol intake for suicidal purposes, the patient was discharged without complications after follow-up for a week ${ }^{5}$. In this case report, hepatotoxicity associated with phenyramidol use recovered fully after drug use was discontinued.

DILI occurs in response to intrinsic and idiosyncratic drug reactions and is generally idiosyncratic in nature. The intrinsic mechanism is predictable, dose-dependent, and characteristic of drug intake at high concentrations, resulting in increased liver injury. DILI may be caused by the drug itself or by a metabolite. Idiosyncratic drug reactions cause hepatotoxicity that is unrelated and unpredictable lag time in susceptible individuals. Approximately $75 \%$ of idiosyncratic drug reactions result in liver transplantation or death. DILI is the most common reason for the discontinuation of an approved drug ${ }^{6}$.

The symptoms of DILI are highly variable, ranging from an asymptomatic elevation in liver enzymes to fulminant liver failure. Biomarkers, such as total bilirubin concentration, AST, ALT, and ALP, can serve as signs of liver injury ${ }^{7}$. Although DILI is not specific, it is based on rates of increase in transaminases and ALP rather than symptoms and signs. The elevation in transaminase and ALP levels and the ratio (R) between basal ALT elevation and basal ALP elevation (ALT / upper limit of normal [ULN]) / (ALP/ULN) determine whether DILI is classified as hepatocellular, cholestatic, or mixed type: hepatocellular DILI is diagnosed when ALT $\geq$ 3-fold ULN (upper limit of normal) and $\mathrm{R} \geq 5$; cholestatic DILI is diagnosed when ALP $\geq 2$-fold ULN and $\mathrm{R} \leq 2$; and mixed DILI is diagnosed when ALT $>3$-fold ULN, ALP $>2$-fold ULN, and $2<\mathrm{R}<5$. The degree of elevation in liver enzymes is poorly correlated with the severity of liver disease. The cholestatic pattern of hepatitis has the lowest mortality, but the liver tests associated with this type require a longer time to normalize. In addition, the cholestatic and mixed patterns of hepatitis are associated with a small but definite risk of evolution to chronicity ${ }^{8,9}$. Our case was evaluated as mixed DILI due to ALT $>3$-fold ULN, ALP $>$ 2-fold 
ULN, and $2<\mathrm{R}<5$ (Table 1 ). To reduce the risk of liver injury caused by drugs, caution should be exercised, especially in older patients, those with chronic diseases, and people with multiple drug use. Liver injury due to drug toxicities occurs more frequently in adults than in children. Patient-associated risk factors for DILI include age, sex, malnutrition, and nutrition $^{10}$.

Our case represents the second case reported in the English literature describing an asymptomatic increase in ALT and AST values due to high-dose phenyramidol intake for the purposes of suicide. Therefore, phenyramidol should be considered a drug that may cause hepatotoxicity. The history of our patient included no reports of recent viral infections, alcohol use, blood transfusions, surgical history, or the chronic use of medication, herbs, or herbal products that might increase the patient's liver enzymes. Therefore, the observed increase in liver enzymes was most likely associated with hepatoxicity due to the use of phenyramidol for a suicide attempt. Various characteristics, such as the absence of any predictable dose-related toxicity associated with phenyramidol, unlike in previously conducted studies, and the absence of hypersensitivity symptoms in our patient, suggested the unique toxicity of this drug. The elevated liver enzymes in our 52-year-old patient returned to normal within 5 days after a suicide attempt associated with the oral intake of high-dose phenyramidol. During cases of asymptomatic ALT and AST elevation, ALP and bilirubin levels are rarely elevated, and severe injury is also extremely rare. If ALT levels rise above 2-fold the upper limit of the reference range, while ALP levels are within the reference range or are only minimally elevated, the DILI should be considered hepatocellular. DILI prevention will require alertness, the determination of patient risk factors, and ALT monitoring for some drugs.

\section{Conclusion}

Drugs are a major cause of liver injury. Drug toxicities can be difficult to diagnose but becomes easier if an agent can be identified that is suspected of causing liver injury. Although DILI is a rare clinical presentation, it has significant morbidity and mortality. Physicians must evaluate other diseases and complications when rapidly diagnosing these patients. Early consultation with a transplantation center is of great importance in addition to identifying the underlying etiology and initiating symptomatic treatment. In this article, we present a case of possible hepatotoxicity associated with the suicidal use of high-dose phenyramidol. Therefore, phenyramidol should be considered as a drug that causes hepatotoxicity.

\section{References}

1. Goodman ZD. Phenotypes and Pathology of Drug-Induced Liver Disease. Clin Liver Dis 2017; 21(1): 89-101. doi: 10.1016/j.cld.2016.08.006. PMID: 27842777.

2. European Association for the Study of the Liver. EASL Clinical Practice Guidelines: Drug-induced liver injury. J Hepatol 2019; 70(6): 1222-61. doi: 10.1016/j.jhep.2019.02.014. PMID: 30926241.

3. Ergün $H$, Polat $O$, Demirkan NA, Günalp $M$, Gürler $S$. The efficacy, safety, and pharmacokinetics of intramuscular and oral phenyramidol in patients with low back pain in an emergency department. Turk J Med Sci 2010; 40(1): 71-6.

4. Köksal AS, Köklü S, Filik L, Sasmaz N, Sahin B. Phenyramidol-associated liver toxicity. Ann Pharmacother 2003; 37(9): 1244-6. doi: 10.1345/aph.1D035. PMID: 12921508.

5. Orak Y, Barçin T, Orak F. Increase of Liver Enzyme Caused by Suicidal Overdose of Phenyramidol Hydrochloride. Journal of the Turkish Society of Intensive Care 2013; 11(2): 80-2.

6. Pandit A, Sachdeva T, Bafna P. Drug-Induced Hepatotoxicity: A Review. Journal of Applied Pharmaceutical Science 2012; 2: 233-43. doi: 10.7324/JAPS.2012.2541.

7. Zárybnický T, Boušová I, Ambrož M, Skálová L. Hepatotoxicity of monoterpenes and sesquiterpenes. Arch Toxicol 2018; 92(1): 1-13. doi: 10.1007/s00204-017-2062-2. PMID: 28905185.

8. Devarbhavi H. An Update on Drug-induced Liver Injury. J Clin Exp Hepatol. 2012;2(3):247-59. doi: 10.1016/j. jceh.2012.05.002. PMID: 25755441; PMCID: PMC3940315.

9. Verma S, Kaplowitz N. Diagnosis, management and prevention of drug-induced liver injury. Gut 2009; 58(11): 1555-64. doi: 10.1136/gut.2008.163675. PMID: 19834119.

10. Shu CC, Lee CH, Lee MC, Wang JY, Yu CJ, Lee LN. Hepatotoxicity due to first-line anti-tuberculosis drugs: a five-year experience in a Taiwan medical centre. Int J Tuberc Lung Dis 2013; 17(7): 934-9. doi: 10.5588/ijtld.12.0782. PMID: 23743313. 